\section{BMJ Paediatrics Open}

\title{
Incidence of Kawasaki disease before and during the COVID-19 pandemic: a retrospective cohort study in Japan
}

Kyohei lio (D) , ${ }^{1}$ Kousaku Matsubara, ${ }^{1}$ Chisato Miyakoshi, ${ }^{2}$ Kunitaka Ota, ${ }^{3}$ Rika Yamaoka, ${ }^{4}$ Junji Eguchi, ${ }^{5}$ Osamu Matsumura, ${ }^{6}$ Takahiro Okutani, ${ }^{7}$ Ikuyo Ueda, ${ }^{8}$ Masahiro Nishiyama ${ }^{9}$

\section{ABSTRACT}

Background Epidemiological studies in Kawasaki disease (KD) have suggested infectious aetiology. During the COVID-19 pandemic, measures for mitigating SARSCoV-2 transmission also suppress the circulation of other contagious microorganisms. The primary objective is to compare the number and incidence of $\mathrm{KD}$ before and during the COVID-19 pandemic in Japan, and the secondary objective is to investigate temporal association between the KD epidemiology and activities of SARSCoV-2 and other viral and bacterial infections.

Methods A retrospective cohort study was conducted between 2016 and 2020 in Kobe, Japan. We collected information of hospitalised KD children in Kobe. Child population was identified through the resident registry system. Activity of COVID-19 and 11 other infectious diseases was derived from a public health monitoring system. Monthly change of KD incidence was analysed using a difference-in-difference regression model. Results Throughout the study period, 1027 KD children were identified. KD had begun to decline in April 2020, coinciding with the beginning of the COVID-19 pandemic. The number of $K D$ cases $(n=66)$ between April and December 2020 was $40 \%$ of the average in the same period in 2016-2019 (165/year). Annual KD incidence was $315,300,353,347$ and 188/100 000 children aged 0-4 years in 2016-2020, respectively. The difference-indifference value of KD incidence was significantly reduced in the fourth quarter in $2020(-15.8,95 \% \mathrm{Cl}-28.0$ to -3.5$)$, compared with that in 2016-2019. Sentinel surveillance showed a marked decrease of all infectious diseases except exanthema subitum after the beginning of the COVID-19 pandemic. There were 86 COVID-19 cases aged $<10$ years and no KD children associated with COVID-19.

Conclusion This study showed that the number and incidence of $\mathrm{KD}$ was dramatically reduced during the COVID-19 pandemic in Japan. This change was temporally associated with decreased activities of various infectious diseases other than COVID-19, supporting the hypothesis of infection-triggered pathogenesis in KD.

\section{INTRODUCTION}

Kawasaki disease $(\mathrm{KD})$ is an acute systemic vasculitis that occurs in young children. ${ }^{1}$ Epidemiological studies describing incidence,

\section{What is known about the subject?}

Continuing nationwide surveys on Kawasaki disease (KD) show that KD incidence has consistently increased since 1970 in Japan.

- Existing research has hypothesised that KD is a consequence of an abnormal immunological response evoked by any of infectious agents in genetically susceptible individuals.

- Measures for avoiding disease transmission during the COVID-19 pandemic, such as use of masks and handwashing, suppress circulation of other contagious microorganisms.

\section{What this study adds?}

The number and incidence of Kawasaki disease (KD) was dramatically decreased to less than $50 \%$ during COVID-19 pandemic, compared with that before the pandemic in Japan.

- The annual KD incidence of $188 / 100000$ children aged $0-4$ years in 2020 was reduced to the nationwide estimates around 2006 in Japan.

- The epidemiological change in KD coincided with decreased activities in various infectious diseases other than COVID-19, supporting the hypothesis of infection-triggered pathogenesis in KD.

age distribution and seasonality in $\mathrm{KD}$ suggest an infectious aetiology. ${ }^{2}{ }^{3}$ However, despite intensive efforts over decades, the aetiology has remained to be elucidated. Some investigators proposed that $\mathrm{KD}$ is a consequence of an abnormal immunological response evoked by any of infectious agents in genetically susceptible individuals, and that these microorganisms may trigger the activation of onset only. $^{2-4}$

Since December 2019, COVID-19 caused by SARS-CoV-2 has been reported globally. ${ }^{5-7}$ Accumulating evidence indicated that children appeared to be less affected and to exhibit milder manifestations than adults. ${ }^{5-7}$ 
However, an exception is the newly emerging, multisystem inflammatory syndrome in children (MIS-C), which shares some clinical and laboratory features with those of $\mathrm{KD}^{8-12}$ Children with MIS-C have been documented mainly in Europe ${ }^{8-10}$ and the USA, ${ }^{11}$ and little is known about MIS-C's prevalence in Asia. Measures for mitigating SARS-CoV-2 transmission have been widely promoted in Japan such as use of masks, handwashing and school closures. These measures may also suppress the circulation of seasonal influenza and other contagious microorganisms, ${ }^{13-16}$ and may reduce $\mathrm{KD}$ under the presumption of its infection-triggered pathogenesis. ${ }^{24}$

These backgrounds raise concerns on an epidemiological association between the number of cases of KD and the magnitude of the COVID-19 pandemic, especially in East Asia where the incidence of $\mathrm{KD}$ is the highest worldwide. ${ }^{1}$ Studies addressing this issue were limited by small sample sizes, being single-centre studies and being evaluated during only the initial stage of the COVID-19 pandemic. ${ }^{17-19}$ In the present study, we conducted a retrospective cohort study in Kobe, Japan. Our primary objective is to compare the number and incidence of KD before and during the COVID-19 pandemic, and secondary objective is to investigate temporal association between the KD epidemiology and activities of SARS-CoV-2 and other viral and bacterial infections.

\section{METHODS}

\section{Study setting and design}

Kobe city is located in west Japan with a population of approximately 1.5 million including approximately 200000 children aged 0-15 years. In 2016, there were 14 hospitals in which children could be hospitalised and treated by paediatricians in Kobe, and four of them had been closed by the end of the study. The study covered all hospitals with paediatric departments in Kobe. Questionnaires were mailed or emailed to representative paediatricians at the 14 hospitals in Kobe and four hospitals in three cities adjacent to Kobe (two hospitals in Akashi and one each in Sanda and Ashiya).

\section{Data collection}

A retrospective cohort study was conducted between 1 January 2016 and 31 December 2020. The surveillance structured form included sex, age in months, date of onset of $\mathrm{KD}$, date of admission, presence or absence of transfer to another institute during treatment and history of COVID-19 within 2 months of KD onset. We also asked the number of hospitalised children with COVID-19 or MIS-C. For cases in which a child with KD appeared twice in the dataset (by readmission within 1 week after the discharge or transfer between two recruiting centres), we retained only one admission in the dataset.

Only patients with KD living in Kobe were included, and the population of children residing in Kobe in each month was identified through the Basic Resident Register system (https://www.city.kobe.lg.jp). To calculate the annual incidence of $\mathrm{KD}$ in children aged $0-4$ years, the total $\mathrm{KD}$ number in each year was divided by the average child population in this age group each month (61 230, 60 216, 58 764, 57 083, and 55172 in 2016, 2017, 2018, 2019 and 2020, respectively). The daily case number of laboratory-confirmed COVID-19 with a 10-year age distribution was obtained from municipal administration information (https://www.city.kobe.lg.jp). The weekly case number of children affected by 11 infectious diseases (influenza, respiratory syncytial virus (RSV) infection, hand-foot-mouth disease, exanthema subitum, herpangina, pharyngoconjunctival fever, group A streptococcal pharyngitis, mumps, erythema infectiosum, infectious gastroenteritis and varicella) was derived from 31 sentinel institutions (Kobe City Infectious Disease Surveillance system: https://kobecity-kmss.jp).

\section{Case definition}

A diagnosis of $\mathrm{KD}$ was based on the Japanese guidelines V.5 or $6 .{ }^{20}{ }^{21}$ We collected data of all patients with complete, incomplete and atypical definite $\mathrm{KD}^{22}{ }^{23}$ The case definition for MIS-C was based on WHO criteria. ${ }^{12}$

\section{Statistics}

Sex distribution was shown as numbers and compared using the $\chi^{2}$ test. Patients' age was shown as median and IQR and compared using the Mann-Whitney U test. $P$ values of $<0.05$ were considered significant. Since it was reported that there is a seasonal change in the incidence of $\mathrm{KD},{ }^{23}$ we used a difference-in-difference regression model to estimate the change in the incidence of KD after the COVID-19 outbreak. For this analysis, we assumed parallel trends and common shocks for each year, and assessed whether the assumptions were held in our data with visual inspection. In this model, the monthly incidence rate of $\mathrm{KD}$ in children aged $0-4$ years was set as the dependent variable. The explanatory variables consisted of a variable for the year of 2020, variables indicating each quarter of the year (January-March, April-June, July-September and October-December) and their interactions. The difference-in-difference value was considered statistically significant if the $95 \%$ CI did not include 0 .

We also constructed a local-level Poisson model with seasonality using the Bayesian method. The model can be described as follows:

$$
\begin{gathered}
\mu[t] \sim \operatorname{Normal}\left(\mu[t-1], \sigma_{m}\right) \\
\log (\lambda[t])=\mu[t]+s_{j}[t]+\log (N[t]) \\
\sum_{j=1}^{12} s_{j}[t]=0 \\
Y[t] \sim \text { Poisson }(\lambda[t])
\end{gathered}
$$

where $Y$ is the number of patients aged $0-4$ years, and the $\log$ of intensity, $\log (\lambda)$, is determined by the random walk component, $\mu$, and the seasonal effect with a zero-sum constraint, $\mathrm{s}_{\mathrm{j}}$, as well as the offset term of population, $\mathrm{N}$. 


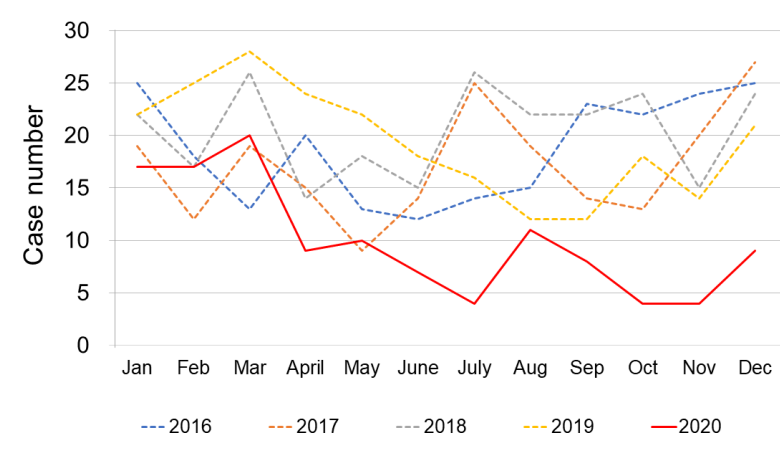

Figure 1 Monthly change of Kawasaki disease cases in 2016-2020.

With this model, we predicted the number of patients with KD using the data before March 2020, then visually compared it with that actually observed. We set four separate sampling chains, each consisting of 12000 samples (including 2000 samples discarded for convergence). We evaluated sampling convergence by Gelman-Rubin statistics (R-hat) and by visually inspecting a trace plot. All statistical analyses were performed by the R software program, V.4.0.2 (R Foundation for Statistical Computing, Vienna, Austria). For Bayesian model analyses, we used the probabilistic programming language Stan (Stan development team).

\section{Patient and public involvement}

There were no patients or public involvement in the research design, process and research findings dissemination.

\section{RESULTS}

Of the 18 eligible facilities, all responded to the survey. A total of $1027 \mathrm{KD}$ children were identified with a male/ female ratio of 579/448. Age of onset ranged from 0 month to 13 years (median: 28 months, IQR: 15-47 months). Figure 1 shows the monthly changes of patients with KD in each year. Seasonality was observed during 2016-2019 with a peak generally in winter (DecemberJanuary) and a reduction in May and June. However, such seasonality disappeared after April 2020, when KD began to decline. The annual number of 120 in 2020 was approximately half (53\%) of the average (227/year) during the previous 4 years. When results were limited to the period between April and December, the reduction rate was more prominent $(60 \%, 66$ in 2020 vs 165 in 2016-2019). On the other hand, distributions of sex (male/female: 513/394 vs 66/54) and age of onset (median age (IQR): 28 (15-47) vs 29 (16-47) months) did not significantly differ between 2016-2019 and 2020.

Notably, the beginning of the decline of KD in 2020 coincided with the onset of a COVID-19 outbreak in Kobe (figure 2): the first adult COVID-19 case was identified on March 2, and the first child aged $<10$ years on April 13. Thereafter, the first spike of the pandemic developed between April and May. Between late May and early June, COVID-19 cases were reduced; however, the second



Figure 2 Daily case numbers of COVID-19 in 2020. Orange and blue bars indicate individuals with $<10$ years and $\geq 10$ years of age, respectively.

and third waves occurred in August and NovemberDecember, respectively (figure 2 ). Since the beginning of pandemic, 86 children aged $<10$ years with COVID-19 were identified, accounting for $2.5 \%$ of the entire cases in 2020. There were 221 COVID-19 cases in the aged 10-19 years population. Of the 86 children aged $<10$ years, 27 children were hospitalised. All were asymptomatic or manifested mild symptoms and were discharged without sequelae. There were no cases of MIS-C and KD following COVID-19 within 2 months.

As previous nationwide surveys reported about $\mathrm{KD}$ in children aged $0-4$ years, we examined changes of $\mathrm{KD}$ in this age group. ${ }^{22}{ }^{23}$ The annual incidence of $\mathrm{KD}$ in children aged $0-4$ years was $315,300,353,347$ and 188 (per 100000 children aged 0-4 years) in 2016, 2017, 2018, 2019 and 2020, respectively. Figure 3 shows the result of a Bayesian time series analysis. We found that patients with $\mathrm{KD}$ had begun to decrease after April 2020, and continued to reduce until December, compared with the prediction on the basis of data before 2020. When limited to the period between April and December, the reduction rate of $\mathrm{KD}$ incidence was $53 \%$ in 2020 compared with the average incidence in 2016-2019 (111 vs $237 / 100000$ children aged $0-4$ years). The result of difference-in-difference analysis is summarised in table 1 . The incidence rate of $\mathrm{KD}$ significantly decreased in the fourth quarter in 2020 compared with 2016 to 2019 $(-15.8,95 \%$ CI $(-28.0$ to -3.5$))$. From visual assessment of figure 1, the assumptions for the difference-in-difference regression model were considered unviolated.

Several practices implemented to contain SARS-CoV-2 transmission have likely had an influence on the activity of other contagious organisms. Sentinel surveillance showed that the annual incidence of 11 infectious diseases, except exanthema subitum, was markedly lower than that in the previous 4 years. Weekly changes of four representative infections (RSV infection, influenza, herpangina and group A streptococcal pharyngitis) in children aged 0-4 years are shown in figure 4 . RSV infections in 2020 were less than one-eighth the average in 2016-2019 (figure 4A). Influenza activity between November and December in 2020 remained at 


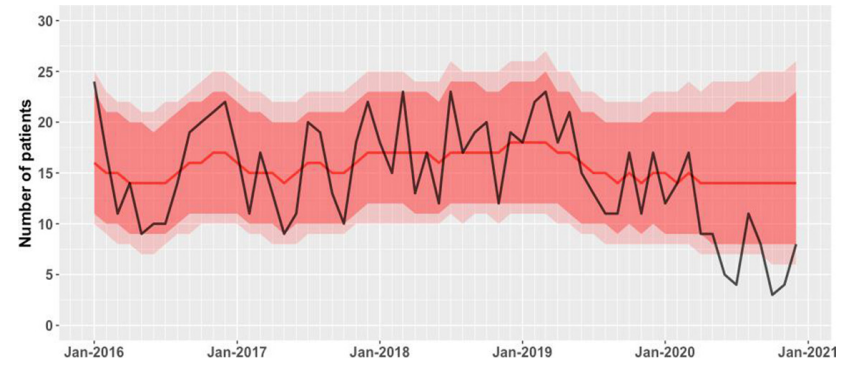

Figure 3 Bayesian time series plot of number of patients with KD aged 0-4 years in 2016-2020. The black line is the observed numbers of patients with KD aged $0-4$, the red line is the predicted median, and the dark and light areas are $90 \%$ and $80 \%$ credible intervals, respectively. KD, Kawasaki disease.

interseasonal levels (figure 4B). In addition, a decrease in case numbers was observed in group A streptococcal pharyngitis (figure 4C) and herpangina (figure 4D).

\section{DISCUSSION}

This retrospective cohort study showed that in Japan KD children in 2020 were dramatically decreased to approximately half of the annual average in 2016-2019. When limited to the COVID-19 pandemic period, the reduction rate was more prominent. These changes were also found in KD incidence among children aged $0-4$ years. A difference-in-difference regression model showed that the incidence in the fourth quarter in 2020 was significantly lower than that in 2016-2019. The KD incidence has shown a consistent increase since 1970 in Japan, which may be reflected by an improved awareness of the diagnosis and possible increase of multiple aetiological agents for $\mathrm{KD} .{ }^{22}{ }^{23}$ The abrupt reduction observed in this study was a marked contrast to this Japanese $\mathrm{KD}$ epidemiology. The KD incidence in 2020 (188/100 000 children aged $0-4$ years) returned to the estimates around $2006 .{ }^{23}$ Notably, the reduction of KD was temporally associated with the COVID-19 pandemic as well as low activities in various infections other than SARS-CoV-2.

The present study has some strengths. It covered all hospitals even small institutes in Kobe and the adjacent three cities with complete response, and the number of children living in Kobe was based on a public registry. Children with $\mathrm{KD}$ are admitted and treated in hospitals
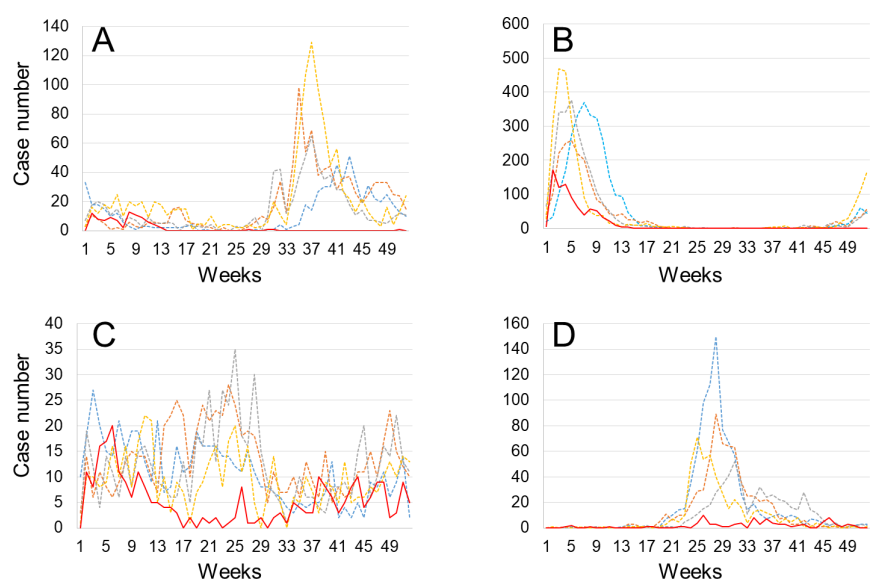

Figure 4 Weekly case numbers of respiratory syncytial virus infection $(A)$, influenza $(B)$, group $A$ streptococcal pharyngitis (C) and herpangina (D) among children aged 0-4 years in 2016-2020. Annual total number of each infection (A-D) was 96, 795, 279 and 94 in 2020, respectively, and $844,2,460,596$ and 629 on average in the years 2016-2019, respectively. Cyan, orange, grey, yellow dotted lines and red solid lines indicate weekly changes in 2016, 2017, 2018, 2019 and 2020, respectively.

in Japan; hence, these approaches allow us to accurately analyse KD incidence. Furthermore, the incidences reported in 2016, 2017 and 2018 in the present study were quite similar to the estimates in Japanese nationwide surveys $^{22}{ }^{23}(315,300$ and 353 vs 309, 317 and 359/100 000 children aged $0-4$ years, respectively), which suggests that our cohort was representative. Simultaneously, we could assess the activity of SARS-CoV-2 and various other pathogens using a public health monitoring system.

$\mathrm{KD}$ has a varied incidence among children of different races and ethnicities with the highest incidence in East Asia, especially in Japan and South Korea. ${ }^{1}$ A seroprevalence study in a single Japanese institute showed no apparent association of COVID-19 and KD. ${ }^{17}$ An early epidemiological analysis in South Korea also failed to detect this association. ${ }^{19}$ We contrarily showed a temporal association between a reduction of KD and the COVID-19 pandemic. The discrepancy between the South Korean study and ours may be reflected by the evaluation period; our study was conducted throughout the year, while the former was limited to the early stage of the COVID-19 pandemic. ${ }^{19}$ We found no MIS-C cases during the

Table 1 Estimated monthly number of Kawasaki disease patients aged 0-4 years and difference-in-difference value between 2020 and $2016-2019$

\begin{tabular}{|c|c|c|c|c|}
\hline & \multicolumn{2}{|c|}{$\begin{array}{l}\text { Estimated monthly no of cases per } 100000 \\
\text { children aged 0-4 years }\end{array}$} & \multirow{2}{*}{\multicolumn{2}{|c|}{$\begin{array}{l}\text { Difference-in-difference value in } 2020 \text { vs } \\
2016-2019(95 \% \mathrm{Cl})\end{array}$}} \\
\hline & 2016-2019 & 2020 & & \\
\hline January-March & 30.2 & 25.6 & & \\
\hline April-June & 22.8 & 13.8 & -4.4 & $(-16.6$ to 7.8$)$ \\
\hline July-September & 26.6 & 14.0 & -8.0 & (-20.2 to 4.2$)$ \\
\hline October-December & 29.6 & 9.2 & -15.8 & $(-28.0$ to -3.5$)$ \\
\hline
\end{tabular}


pandemic, in line with the other studies in $\operatorname{Japan}^{17}$ and South Korea. ${ }^{24}$ This may be explained by recent findings that MIS-C shows a predilection for individuals of black or Hispanic descent. ${ }^{12}{ }^{25} 26$ In the USA, as of 20 February 2021, 2060 MIS-C cases were identified while COVID-19 cases in children aged 0-17 years were up to 2.39 million. ${ }^{26}{ }^{27}$ In contrast, because of small number of COVID-19 cases ( $n=307)$ in the patients under 20 years of age in this study, we were not able to conclude that MIS-C was rare in Japanese children. A larger study is warranted to definitively assess these associations in Japan.

The exact reasons of the significant reduction of $\mathrm{KD}$ are unclear. It seems to be unlikely that SARS-CoV-2 itself directly suppressed the occurrence of $\mathrm{KD}$, because only a small number of children aged $<10$ years were affected with this virus in Kobe. The most plausible explanation is that various measures to reduce the spread of SARS-CoV-2 also suppressed the transmission activities of other microorganisms, and thereby reduced KD. ${ }^{13} 14$ Weekly epidemics of 11 infectious diseases in Kobe paralleled nationwide surveillance reports. ${ }^{28}$ The disappearance of seasonality in KD in 2020 provides evidence for the contribution of multiple viruses to $\mathrm{KD}$ pathogenesis. Taken together, our findings support the hypothesis of infection-triggered pathogenesis in KD.

Several limitations are included this study. First, our findings indicated a temporal association between the decline in KD and the increase of SARS-CoV-2-affected individuals as well as a reduction of various infections. However, studies evaluating epidemiological time series could not fully account for this causality. Numerous infectious agents not examined in this study or other factors, such as environmental and behavioural changes, may contribute to KD occurrence. Second, our study was conducted in Japan. Whether similar findings are generalised to other regions with different genetic ethnicities, magnitude of COVID-19 pandemic, and protective measures remains to be determined.

\section{CONCLUSION}

The present study showed that KD significantly decreased during the COVID-19 pandemic in Kobe, Japan. Epidemiological analysis through the public surveillance system also showed a profound decrease of various types of infections, probably due to the implementation of protective measures against SARS-CoV-2 transmission. Our data support, at least in part, the hypothesis that many microorganisms are involved to trigger $\mathrm{KD}$ occurrence.

\footnotetext{
Author affiliations

${ }^{1}$ Department of Pediatrics, Kobe City Nishi-Kobe Medical Center, Kobe, Hyogo, Japan

${ }^{2}$ Department of Pediatrics, Kobe City Medical Center General Hospital, Kobe, Hyogo, Japan

${ }^{3}$ Department of Pediatrics, Konan Medical Center, Kobe, Hyogo, Japan ${ }^{4}$ Department of Pediatrics, National Hospital Organization Kobe Medical Center, Kobe, Hyogo, Japan
}

${ }^{5}$ Department of Pediatrics, Kobe City Medical Center West Hospital, Kobe, Hyogo, Japan

${ }^{6}$ Department of General Pediatrics, Hyogo Prefectural Kobe Children's Hospital, Kobe, Hyogo, Japan

${ }^{7}$ Department of Pediatrics, Saiseikai Hyogo-ken Hospital, Kobe, Hyogo, Japan ${ }^{8}$ Department of Pediatrics, Japan Community Health Care Organization Kobe Central Hospital, Kobe, Hyogo, Japan

${ }^{9}$ Department of Pediatrics, Kobe University Graduate School of Medicine, Kobe, Hyogo, Japan

Acknowledgements The authors appreciate S. Nukina (Department of Pediatrics, Akashi Municipal Hospital), N. Yokoyama (Department of Pediatrics, Akashi Medical Center), A. Hirase (Department of Pediatrics, Kumon Hospital), T. Inoue (Department of Pediatrics, Sanda City Hospital), T. Morita (Department of Pediatrics, Ashiya Municipal Hospital), Y. Matsuura (Kobe Tokushukai Hospital), H. Yamashita (Director of hospital, Kobe Red Cross Hospital) and K. Tsukuda (Department of Pediatrics, Mahoshi Hospital), for providing the clinical records of the patients.

Contributors $\mathrm{KM}$ and $\mathrm{CM}$ conceptualised and designed the study. $\mathrm{KI}, \mathrm{KM}$ and $\mathrm{CM}$ drafted the manuscript. CM carried out statistical analyses. All authors collected the data and critically reviewed the manuscript. All authors read and approved the final manuscript as submitted.

Funding The authors have not declared a specific grant for this research from any funding agency in the public, commercial or not-for-profit sectors.

Competing interests None declared.

Patient and public involvement Patients and/or the public were not involved in the design, or conduct, or reporting, or dissemination plans of this research.

Patient consent for publication Not required.

Ethics approval The study was approved by the Ethics Committee of Kobe City Nishi-Kobe Medical Center (receipt ID: 2020-37). Review board approval in each institution was not necessarily required for the assessment of anonymised surveillance.

Provenance and peer review Not commissioned; externally peer reviewed.

Data availability statement Data are available on reasonable request. KI and KM had full access to the data in the study and take responsibility for the accuracy and integrity of the data. Data are available on reasonable request.

Open access This is an open access article distributed in accordance with the Creative Commons Attribution Non Commercial (CC BY-NC 4.0) license, which permits others to distribute, remix, adapt, build upon this work non-commercially, and license their derivative works on different terms, provided the original work is properly cited, appropriate credit is given, any changes made indicated, and the use is non-commercial. See: http://creativecommons.org/licenses/by-nc/4.0/.

ORCID iD

Kyohei lio http://orcid.org/0000-0002-7356-5307

\section{REFERENCES}

1 Burns JC, Glodé MP. Kawasaki syndrome. Lancet 2004;364:533-44.

2 Principi N, Rigante D, Esposito S. The role of infection in Kawasaki syndrome. J Infect 2013;67:1-10.

3 Rowley AH. Is Kawasaki disease an infectious disorder? Int $J$ Rheum Dis 2018;21:20-5.

4 Nagao $\mathrm{Y}$, Urabe $\mathrm{C}$, Nakamura $\mathrm{H}$, et al. Predicting the characteristics of the aetiological agent for Kawasaki disease from other paediatric infectious diseases in Japan. Epidemiol Infect 2016;144:478-92.

5 Swann OV, Holden KA, Turtle L, et al. Clinical characteristics of children and young people admitted to hospital with covid-19 in United Kingdom: prospective multicentre observational cohort study. BMJ 2020;370:m3249.

6 Wald ER, Schmit KM, Gusland DY. A pediatric infectious disease perspective on COVID-19. Clin Infect Dis 2020:ciaa1095.

7 Viner RM, Mytton OT, Bonell C, et al. Susceptibility to SARSCoV-2 infection among children and adolescents compared with adults: a systematic review and meta-analysis. JAMA Pediatr 2021;175:e204573.

8 Riphagen S, Gomez X, Gonzalez-Martinez C, et al. Hyperinflammatory shock in children during COVID-19 pandemic. Lancet 2020;395:1607-8. 
9 Verdoni L, Mazza A, Gervasoni A, et al. An outbreak of severe Kawasaki-like disease at the Italian epicentre of the SARS-CoV-2 epidemic: an observational cohort study. Lancet 2020;395:1771-8.

10 Toubiana J, Poirault C, Corsia A, et al. Kawasaki-like multisystem inflammatory syndrome in children during the covid-19 pandemic in Paris, France: prospective observational study. BMJ 2020;369:m2094.

11 Feldstein LR, Rose EB, Horwitz SM, et al. Multisystem inflammatory syndrome in U.S. children and adolescents. N Engl J Med 2020;383:334-46.

12 Jiang L, Tang K, Levin M, et al. COVID-19 and multisystem inflammatory syndrome in children and adolescents. Lancet Infect Dis 2020;20:e276-88.

13 Chu DK, Akl EA, Duda S, et al. Physical distancing, face masks, and eye protection to prevent person-to-person transmission of SARS-CoV-2 and COVID-19: a systematic review and meta-analysis. Lancet 2020;395:1973-87.

14 Viner RM, Russell SJ, Croker $\mathrm{H}$, et al. School closure and management practices during coronavirus outbreaks including COVID-19: a rapid systematic review. Lancet Child Adolesc Health 2020;4:397-404.

15 Sakamoto $\mathrm{H}$, Ishikane $\mathrm{M}$, Ueda $\mathrm{P}$. Seasonal influenza activity during the SARS-CoV-2 outbreak in Japan. JAMA 2020;323:1969-71.

16 WHO. Influenza update $\mathrm{N}^{\circ} 380$. Available: https://www.who.int/ publications/m/item/influenza-update-n-380

17 lio K, Uda K, Hataya H, et al. Kawasaki disease or Kawasaki-like disease: influence of SARS-CoV-2 infections in Japan. Acta Paediatr 2021;110:600-1.

18 Bordet J, Perrier S, Olexa C, et al. Paediatric multisystem inflammatory syndrome associated with COVID-19: filling the gap between myocarditis and Kawasaki? Eur J Pediatr 2021;180:877-84.
19 Choe S-A, An HS, Choe YJ. No temporal association between human coronavirus and Kawasaki disease: national data from South Korea. J Med Virol 2021;93:585-7.

20 Ayusawa M, Sonobe T, Uemura S, et al. Revision of diagnostic guidelines for Kawasaki disease (the 5 th revised edition). Pediatr Int 2005;47:232-4.

21 Kobayashi T, Ayusawa M, Suzuki H, et al. Revision of diagnostic guidelines for Kawasaki disease (6th revised edition). Pediatr Int 2020;62:1135-8.

22 Makino N, Nakamura Y, Yashiro M, et al. Nationwide epidemiologic survey of Kawasaki disease in Japan, 2015-2016. Pediatr Int 2019;61:397-403.

23 Ae R, Makino N, Kosami K, et al. Epidemiology, treatments, and cardiac complications in patients with Kawasaki disease: the nationwide survey in Japan, 2017-2018. J Pediatr 2020;225:23-9.

24 Kim YJ, Park H, Choi YY, et al. Defining association between COVID-19 and the multisystem inflammatory syndrome in children through the pandemic. J Korean Med Sci 2020;35:e204.

25 Rowley $\mathrm{AH}$. Multisystem inflammatory syndrome in children and Kawasaki disease: two different illnesses with overlapping clinical features. J Pediatr 2020;224:129-32.

26 Health Department-Reported cases of multisystem inflammatory syndrome in children (MIS-C) in the United States /CDC. Available: https://www.cdc.gov/mis-c/cases/index.html [Accessed 20 Feb 2021].

27 CDC. COVID data Tracker. Available: https://covid.cdc.gov/coviddata-tracker/\#demographics [Accessed 20 Feb 2021].

28 National Institutes Infectious Diseases. Infectious diseases Weekly report. Available: https://www.niid.go.jp/niid/ja/idwr.html [Accessed 15 Jan 2021] 